ELECTRONIC RESEARCH ANNOUNCEMENTS OF THE AMERICAN MATHEMATICAL SOCIETY

Volume 3, Pages 11-16 (March 12, 1997)

S $1079-6762(97) 00018-8$

\title{
ERGODIC BEHAVIOR OF GRAPH ENTROPY
}

\author{
JOHN KIEFFER AND EN-HUI YANG
}

(Communicated by Douglas Lind)

\begin{abstract}
For a positive integer $n$, let $X^{n}$ be the vector formed by the first $n$ samples of a stationary ergodic finite alphabet process. The vector $X^{n}$ is hierarchically represented via a finite rooted acyclic directed graph $G_{n}$. Each terminal vertex of $G_{n}$ carries a label from the process alphabet, and $X^{n}$ can be reconstituted as the sequence of labels at the ends of the paths from root vertex to terminal vertex in $G_{n}$. The entropy $H\left(G_{n}\right)$ of the graph $G_{n}$ is defined as a nonnegative real number computed in terms of the number of incident edges to each vertex of $G_{n}$. An algorithm is given which assigns to $G_{n}$ a binary codeword from which $G_{n}$ can be reconstructed, such that the length of the codeword is approximately equal to $H\left(G_{n}\right)$. It is shown that if the number of edges of $G_{n}$ is $o(n)$, then the sequence $\left\{H\left(G_{n}\right) / n\right\}$ converges almost surely to the entropy of the process.
\end{abstract}

\section{INTRODUCTION}

In the hierarchical approach to data compression developed by the authors [2], [3], [4], finite rooted acyclic directed graphs can be used to represent the data strings that are to be compressed. To see how this representation works, let us determine the data string $x$ represented by the graph in Figure 1. This graph contains ten edges labelled 1 through 10 and two terminal vertices labelled 0 and 1 . If we list the paths in this graph that go from root vertex to a terminal vertex, we obtain the ten paths

$$
\text { (1), }(2,5),(2,6),(3,7,5),(3,7,6),(3,8),(4,9,7,5),(4,9,7,6),(4,9,8),(4,10) .
$$

(The paths are listed in lexicographical order.) The string $x$ is then obtained by replacing each path in this list with the label on the terminal vertex for that path. We see that $x=0010110111$.

Let $G=(V, E)$ denote an arbitrary finite rooted acyclic directed graph, where $V$ is the set of vertices and $E$ is the set of edges. Let $|V|,|E|$ denote the number of vertices and the number of edges, respectively. (In general, let $|S|$ denote the cardinality of any finite set $S$.) For each $v \in V$, let $i(v)$ be the number of edges

Received by the editors December 12, 1996.

1991 Mathematics Subject Classification. Primary 28D99; Secondary 60G10, 94A15.

Key words and phrases. Graphs, entropy, compression, stationary ergodic process.

This work was supported in part by the National Science Foundation under Grants NCR9304984 and NCR-9627965. 


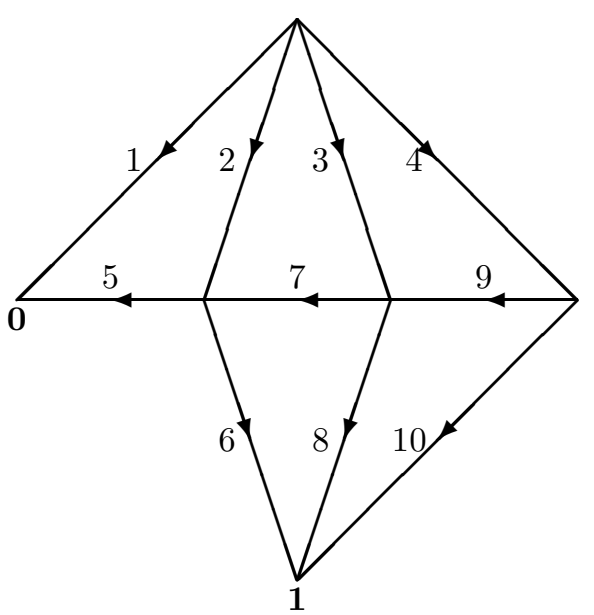

Figure 1

that terminate at $v$. Following [2], the entropy $H(G)$ of the graph $G$ is defined by

$$
H(G)=\sum_{v \in V, i(v) \geq 2}(i(v)-1) \log \left(\frac{|E|-|V|+1}{i(v)-1}\right),
$$

where the logarithm throughout shall be to base two. The entropy of the graph in Figure 1 is $3 \log 5+2 \log (5 / 2)=9.61$.

Suppose we are given an infinite finite-alphabet sequence $\left(x_{1}, x_{2}, \ldots\right)$, and let $x^{n}=\left(x_{1}, x_{2}, \ldots, x_{n}\right), n \geq 1$. For each $n$, a graph $G_{n}$ is selected to represent the data string $x^{n}$. One then compresses $x^{n}$ by assigning a binary codeword to $G_{n}$ that allows one to reconstruct $G_{n}$ and therefore $x^{n}$. The length of this codeword is approximately equal to $H\left(G_{n}\right)$. (See Lemma 1.) A natural question is the behavior of the entropies $\left\{H\left(G_{n}\right)\right\}$ as $n \rightarrow \infty$ when the sequence $\left(x_{1}, x_{2}, \ldots\right)$ is generated by a stationary ergodic process. In this case, our main result (Theorem 1) states that $\left\{H\left(G_{n}\right) / n\right\}$ converges almost surely to the entropy of the process, provided only that the number of edges of $G_{n}$ is $o(n)$. Some of the many applications of this result shall be discussed.

\section{MAin RESUlt}

If $B$ is a finite set, let $B^{*}$ denote the set of all strings of finite length formed from symbols in $B$. Suppose $s_{1}, s_{2}, \ldots, s_{n}$ are strings in a set $B^{*}$. Let $s_{1} * s_{2} * \cdots * s_{n}$ denote the string in $B^{*}$ obtained by concatenating together the strings $s_{1}, s_{2}, \ldots, s_{n}$ in the indicated order.

Let $G=(V, E)$ be a finite rooted acyclic directed graph. Let $V_{t}$ denote the set of terminal vertices of $G$. For each $v \in V, v \notin V_{t}$, let $E^{+}(v)$ denote the set of all edges emanating from $v$. For each $v \in V, v \neq$ root vertex, let $E^{-}(v)$ denote the set of all edges which terminate at $v$. We say that $G$ is a canonical graph if

(i): $V=\{1,2, \ldots,|V|\}$ and $1 \in V$ is the root vertex.

(ii): $E=\{1,2, \ldots,|E|\}$.

(iii): If $v_{1}, v_{2} \in\{2, \ldots,|V|\}$ and $v_{1}<v_{2}$, then $\min E^{-}\left(v_{1}\right)<\min E^{-}\left(v_{2}\right)$.

(iv): If $v_{1}, v_{2} \in\left\{v \in V: v \notin V_{t}\right\}$ and $v_{2}>v_{1}$, then $\min E^{+}\left(v_{2}\right)>\max E^{+}\left(v_{1}\right)$. 
Every finite acyclic rooted directed graph is isomorphic to a canonical graph, and graph entropy is an isomorphism invariant; therefore, we concentrate on canonical graphs from now on.

Let $G=(V, E)$ be a finite rooted acyclic directed canonical graph. There is a unique mapping $\psi_{G}: V \rightarrow V_{t}^{*}$ satisfying the following two rules:

(i): $\psi_{G}(v)=v, v \in V_{t}$.

(ii): If $v \in V, v \notin V_{t}, r=\min E^{+}(v)$, and $s=\max E^{+}(v)$, then

$$
\psi_{G}(v)=\psi_{G}(v(r)) * \psi_{G}(v(r+1)) * \cdots * \psi_{G}(v(s)),
$$

where $v(e)$ denotes the vertex at which edge $e$ terminates.

Let $\left(v_{1}, v_{2}, \ldots, v_{k}\right)$ be the string $\psi_{G}(1)$. If $\left(x_{1}, x_{2}, \ldots, x_{k}\right)$ is a string of the same length, whose symbols $\left\{x_{i}\right\}$ are selected from any set whatsoever, we write $G \rightarrow x$ if there is a one-to-one mapping $f: V_{t} \rightarrow\left\{x_{1}, x_{2}, \ldots, x_{k}\right\}$ such that $x_{i}=f\left(v_{i}\right)$ for $1 \leq i \leq k$. Let $\mathcal{G}$ denote the set of all finite rooted acyclic directed canonical graphs $G$ such that $\psi_{G}$ is one-to-one.

We fix a finite nonempty set $A$ for the rest of the paper.

Theorem 1. For each $x \in A^{*}$, let $G(x)=(V(x), E(x))$ be a graph in $\mathcal{G}$ such that $G(x) \rightarrow x$. Let $\left(X_{1}, X_{2}, \ldots\right)$ be an A-valued stationary ergodic process with entropy $H$. Assume that

$$
\left|E\left(X_{1}, X_{2}, \ldots, X_{n}\right)\right| / n \rightarrow 0 \text { almost surely as } n \rightarrow \infty .
$$

Then

$$
H\left(G\left(X_{1}, X_{2}, \ldots, X_{n}\right)\right) / n \rightarrow H \text { almost surely as } n \rightarrow \infty \text {. }
$$

\section{Applications}

1. For each $x \in A^{*}$, let $G(x)=(V(x), E(x))$ be a graph in $\mathcal{G}$ such that $G(x) \rightarrow x$. Suppose that $\max \left\{|E(x)|: x \in A^{n}\right\}=o(n)$. As shown in [2], there is a computationally attractive data compression algorithm that assigns to each sufficiently long $x \in A^{*}$ a binary codeword of length approximately equal to $H(G(x))$. Theorem 1 tells us that this algorithm optimally compresses the first $n$ data samples generated by any stationary ergodic $A$-valued process, asymptotically as $n \rightarrow \infty$. ("Optimally compresses" refers to the well-known fact [1] that no compression algorithm can achieve an asymptotic compression rate in code bits per data sample less than the entropy of the process generating the data samples.)

2. The well-known Lempel-Ziv parsing rule [5] partitions each string $x \in A^{*}$ into $t=t(x)$ phrases such that

(i): Each phrase is either a singleton or is obtained by adjoining a symbol to the end of a preceding phrase.

(ii): The first $t-1$ phrases are distinct.

For example, the Lempel-Ziv parsing of the data string 0010110111 is (0), (01), (011), (0111). Let $\left(X_{1}, X_{2}, \ldots\right)$ be an $A$-valued stationary ergodic process with entropy $H$. Theorem 1 can be used to deduce the asymptotic expansion

$$
t\left(X_{1}, X_{2}, \ldots, X_{n}\right)=\frac{H n}{\log n}+o\left(\frac{n}{\log n}\right) \text { almost surely. }
$$

(One defines a graph $G(x) \rightarrow x$ such that $H(G(x))$ is approximately equal to $t(x) \log n$ whenever $x \in A^{n}$ and $n$ is large.) 
3. Let $\phi:[0, \infty) \rightarrow(-\infty, \infty)$ be the function such that $\phi(0)=0$ and $\phi(x)=x \log x$ for $x>0$. Let $n>1$ be an integer and let $x \in A^{2^{n}}$. For each integer $k$ such that $0 \leq k \leq n$, let $S_{k}(x)$ be the set of all $y \in A^{2^{k}}$ that appear in the partitioning of $x$ into substrings of length $2^{k}$. If $y \in A^{2^{k}}$ for $0 \leq k<n$, let $N_{l}(y \mid x)$ be the number of $z$ such that $y * z \in S_{k+1}(x)$ and let $N_{r}(y \mid x)$ be the number of $z$ such that $z * y \in S_{k+1}(x)$. Define $Q_{k}(x)$ to be the number

$$
Q_{k}(x)=\phi\left(\sum_{y \in A^{2^{k}}}\left\{N_{l}(y \mid x)+N_{r}(y \mid x)-1\right\}\right)-\sum_{y \in A^{2^{k}}} \phi\left(N_{l}(y \mid x)+N_{r}(y \mid x)-1\right) .
$$

Let $\left(X_{1}, X_{2}, \ldots\right)$ be an $A$-valued stationary ergodic process with entropy $H$. Using Theorem 1 one can deduce the following limit formula for $H$ :

$$
\frac{1}{2^{n}} \sum_{k=0}^{n-1} Q_{k}\left(X_{1}, X_{2}, \ldots, X_{2^{n}}\right) \rightarrow H \text { almost surely as } n \rightarrow \infty .
$$

Each string $y$ lying in the union of the $S_{k}(x), 0 \leq k \leq n$, generates a vertex $v(y)$ of a graph $G(x)$. If the length of $y$ is at least two, two edges emanate from $v(y)$, one going to $v\left(y_{1}\right)$ and one going to $v\left(y_{2}\right)$, where $y_{1}$ and $y_{2}$ are the right and left halves of $y$. One then applies Theorem 1 to the graphs $\{G(x)\}$.

\section{Ancillary Results}

Lemma 1. Let $k$ be a positive integer. Let $\mathcal{G}_{k}=\{G=(V, E) \in \mathcal{G}:|E| \leq k\}$. The members of $\mathcal{G}_{k}$ can be assigned distinct binary codewords so that

(i): The codeword assigned to $G \in \mathcal{G}_{k}$ is of length no greater than $H(G)+6 k+1$.

(ii): No codeword is a prefix of any other codeword.

Lemma 2. For $0<\epsilon<1$, and $n$ sufficiently large, define $h_{\epsilon}(x)$ for $x \in A^{n}$ by

$$
h_{\epsilon}(x)=\min \{H(G): G=(V, E) \in \mathcal{G}, G \rightarrow x,|E|<n \epsilon\} .
$$

Then

$$
\lim _{\epsilon \rightarrow 0} \limsup _{n \rightarrow \infty} \frac{1}{n} \log \left(\sum_{x \in A^{n}} 2^{-h_{\epsilon}(x)}\right)=0 .
$$

Lemma 3. Let $k$ be a positive integer. Then there exists a function $f_{k}:(0, \infty) \rightarrow$ $(-\infty, \infty)$ satisfying $\lim _{t \rightarrow 0^{+}} f_{k}(t)=0$ for which

$$
H(G) / n \leq f_{k}(|E| / n)-\frac{1}{n k} \sum_{i=0}^{n-k+1} \log \mu\left(x_{i}, x_{i+1}, \ldots, x_{i+k-1}\right)
$$

whenever $n \geq k, \mu$ is a probability distribution on $A^{k}, x \in A^{n}$, and $G=(V, E) \in \mathcal{G}$ with $G \rightarrow x$.

\section{Proofs}

Proof of Lemma 1. We call the binary symbols in the codeword for $G=(V, E) \in \mathcal{G}_{k}$ "codebits". The first $6 k$ codebits in the codeword serve to identify each of the following six entities concerning $G$ :

(i): $|E|$.

(ii): $|V|$.

(iii): $V_{t}$. 
(iv): The cardinalities of the sets $E^{+}(v), v \in V, v \notin V_{t}$.

(v): The cardinalities of the sets $E^{-}(v), v \in V, v \neq 1$.

(vi): The positions in the vector $(v(1), v(2), \ldots, v(|E|))$ where each vertex $v \neq$ $1 \in V$ first appears.

Let $s_{G}$ be the string obtained from $(v(1), v(2), \ldots, v(|E|))$ by deleting the first appearance of each vertex $v \neq 1 \in V$. The $J$ remaining codebits identify the string $s_{G}$, and one then identifies $G$ from $s_{G}$ and items (i)-(vi). The string $s_{G}$ lies in the set $S_{G}$ of all strings of length $|E|-|V|+1$ in which each $v \neq 1 \in V$ appears $\left|E^{-}(v)\right|-1$ times. Taking $J=\left\lceil\log \left|S_{G}\right|\right\rceil, J \leq H(G)+1$.

Proof of Lemma 2. Fix $n$ so large that $h_{\epsilon}(x)$ is defined for each $x \in A^{n}$. For each such $x$, pick a $G_{x}=\left(V_{x}, E_{x}\right) \in \mathcal{G}$ such that $H\left(G_{x}\right)=h_{\epsilon}(x), G_{x} \rightarrow x$, and $\left|E_{x}\right|<n \epsilon$. According to Lemma 1, we can assign to each $G \in \mathcal{G}_{n}=\left\{G_{x}: x \in A^{n}\right\}$ a binary codeword of length $L(G) \leq H(G)+6 n \epsilon+1$. Kraft's inequality from information theory ([1], page 82) tells us that $\sum_{G \in \mathcal{G}_{n}} 2^{-L(G)} \leq 1$. From this and the fact that there are $\leq|A|^{n \epsilon}$ strings $x \in A^{n}$ such that $G \rightarrow x$ for each $G \in \mathcal{G}_{n}$, it follows that

$$
\sum_{x \in A^{n}} 2^{-L\left(G_{x}\right)} \leq|A|^{n \epsilon}
$$

and therefore

$$
\sum_{x \in A^{n}} 2^{-h_{\epsilon}(x)} \leq 2^{6 n \epsilon+1}|A|^{n \epsilon}
$$

Proof of Lemma 3. Fix $k$ and a probability distribution $\mu$ on $A^{k}$. For each pair $j, n$ in which $0 \leq j<k$ and $n \geq k$, let $W_{j, n}=\{1 \leq i \leq n-k+1: i \equiv j \bmod k\}$. For each string $s=\left(s_{1}, s_{2}, \ldots, s_{n}\right) \in A^{*}$, define $\lambda(s)$ as follows:

(i): $\lambda(s)=1, n<k$.

(ii): $\lambda(s)=\max _{0 \leq j<k}\left[\prod_{i \in W_{j, n}} \mu\left(s_{i}, s_{i+1}, \ldots, s_{i+k-1}\right)\right], n \geq k$,

where an empty product is taken to be one. For each $s \in A^{*}$, define $\lambda^{*}(s)=$ $C^{-1}|s|^{-2} \lambda(s)$, where $|s|$ denotes the length of $s$ and $C$ is the positive real constant (depending on $k$ ) that makes the numbers $\left\{\lambda^{*}(s): s \in A^{*}\right\}$ sum to one. Fix $n \geq k, x=\left(x_{1}, \ldots, x_{n}\right) \in A^{n}$, and $G=(V, E) \in \mathcal{G}$ such that $G \rightarrow x$. Let $r=|E|-|V|+\left|V_{t}\right|+1$. The function which carries $\psi_{G}(1)$ into $x$ also carries each $\psi_{G}(v)$ into a string $\psi_{G}^{*}(v)(v \in V, v \neq 1)$. There exist strings $\left\{s_{i}: 1 \leq i \leq r\right\}$ such that

(i): $\left\{s_{i}:|E|-|V|+1<i \leq r\right\}=\left\{x_{1}, x_{2}, \ldots, x_{n}\right\}$.

(ii): $\left|\left\{1 \leq i \leq|E|-|V|+1: s_{i}=\psi_{G}^{*}(v)\right\}\right|=\left|E^{-}(v)\right|-1, v \in V, v \neq 1$.

(iii): $x=\tilde{s}_{1} * \tilde{s}_{2} * \cdots * \tilde{s}_{r}$, for some permutation $\left\{\tilde{s}_{i}\right\}$ of $\left\{s_{i}\right\}$.

Note that

$$
\prod_{i=1}^{n-k+1} \mu\left(x_{i}, \ldots, x_{i+k-1}\right) \leq\left[\prod_{i=1}^{r} \lambda\left(s_{i}\right)\right]^{k} .
$$

Replacing $\lambda\left(s_{i}\right)$ by $C\left|s_{i}\right|^{2} \lambda^{*}\left(s_{i}\right)$, and taking the logarithm of both sides,

$$
-\sum_{i=1}^{|E|-|V|+1} \log \lambda^{*}\left(s_{i}\right) \leq 2 \sum_{i=1}^{r} \log \left|s_{i}\right|+r \log C-\frac{1}{k} \sum_{i=1}^{n-k+1} \log \mu\left(x_{i}, \ldots, x_{i+k-1}\right) .
$$


Concavity of the logarithm function yields the bound

$$
2 \sum_{i=1}^{r} \log \left|s_{i}\right| \leq 2 r \log \left(\frac{n}{r}\right) .
$$

For each $s=\psi_{G}^{*}(v), v \in V, v \neq 1$, define $\sigma(s)=\left(\left|E^{-}(v)\right|-1\right) /(|E|-|V|+1)$. Then

$$
H(G)=-\sum_{i=1}^{|E|-|V|+1} \log \sigma\left(s_{i}\right) \leq-\sum_{i=1}^{|E|-|V|+1} \log \lambda^{*}\left(s_{i}\right) .
$$

Condition (4.1) is true with $f_{k}(t)=\sup _{0<\delta \leq t}\{\delta \log C-2 \delta \log \delta\}$.

Proof of Theorem 1. Let $\left(X_{1}, X_{2}, \ldots\right)$ be a stationary ergodic $A$-valued process with entropy $H$. For $n \geq 1$, let $X^{n}=\left(X_{1}, X_{2}, \ldots, X_{n}\right)$. If $x \in A^{*}$ has length $n$, define $\mu(x)=\operatorname{Pr}\left[X^{n}=x\right]$. From (4.1) we see that $\lim _{\sup } \operatorname{sum}_{n \rightarrow \infty} H\left(G\left(X^{n}\right)\right) / n \leq H$ almost surely.

Fix $\delta>0$. By Lemma 2, there exists $\epsilon$ such that for $n$ sufficiently large,

$$
\operatorname{Pr}\left[\log \left(\frac{2^{-h_{\epsilon}\left(X^{n}\right)}}{\mu\left(X^{n}\right)}\right) \geq n \delta\right]<2^{-n \delta / 2} .
$$

Applying the Borel-Cantelli lemma,

$$
\limsup _{n \rightarrow \infty} \frac{1}{n} \log \left(\frac{2^{-h_{\epsilon}\left(X^{n}\right)}}{\mu\left(X^{n}\right)}\right) \leq \delta \text { almost surely. }
$$

Replacing $h_{\epsilon}\left(X^{n}\right)$ by $H\left(G\left(X^{n}\right)\right)$ in (5.1), and then using the fact that

$$
-n^{-1} \log \mu\left(X^{n}\right) \rightarrow H \text { a.s. }
$$

(Shannon-McMillan-Breiman Theorem), one concludes that

$$
\liminf _{n \rightarrow \infty} H\left(G\left(X^{n}\right)\right) / n \geq H-\delta \text { almost surely, }
$$

for an arbitrary $\delta>0$.

\section{REFERENCES}

[1] T. M. Cover and J. A. Thomas, Elements of information theory, Wiley, New York, 1991. MR 92g:94001

[2] J. Kieffer and E.-H. Yang, "A complexity reduction method for lossless source code design," Technical Report, Dept. of Electrical Engineering, University of Minnesota Twin Cities, 1995 (http://www.ee.umn.edu/users/kieffer).

[3] J. Kieffer, E.-H. Yang, G. Nelson, and P. Cosman, "Lossless compression via bisection trees," Technical Report, Dept. of Electrical Engineering, University of Minnesota Twin Cities, 1996 (http://www.ee.umn.edu/users/kieffer).

[4] J. Kieffer, G. Nelson, and E.-H. Yang, "Tutorial on the quadrisection method for lossless data compression," Technical Report, Dept. of Electrical Engineering, University of Minnesota Twin Cities, 1996 (http://www.ee.umn.edu/users/kieffer).

[5] A. Lempel and J. Ziv, On the complexity of finite sequences, IEEE Trans. Inform. Theory, 22 (1976), 75-81. MR 52:10234

Department of Electrical Engineering, University of Minnesota, 200 Union Street SE, Minneapolis, MN 55455

E-mail address: kieffer@ee.umn.edu

Department of Mathematics, Nankai University, Tianjin 300071, P. R. China

E-mail address: ehyang@irving.usc.edu 